\title{
Algoritmo Para Resolver Operaciones Combinadas Con Números Reales
}

\author{
Leonidas Cerda, Doctor \\ Martha Dávalos, Matemático \\ Janneth Morocho, Matemático \\ Escuela Superior Politécnica de Chimborazo, Ecuador
}

Doi: 10.19044/esj.2018.v14n33p24 URL:http://dx.doi.org/10.19044/esj.2018.v14n33p24

\begin{abstract}
In arithmetic with real numbers, it is essential that students hierarchize the operations to reduce arithmetic polynomials. The domain of these operations forms the basis for understanding other mathematical structures that are studied throughout the student's life. Building the practice of this knowledge will facilitate future learning in the area of mathematics. In this research it was proved that the use of an appropriate algorithm to realize operations with real numbers positively affects the learning of the students who enter the Higher Polytechnic School of Chimborazo, Faculty of Natural Resources, Agronomy Career.
\end{abstract}

Keywords: Algorithm, Operations, Real

\section{Resumen}

En la aritmética con números reales, es primordial que los estudiantes jerarquicen las operaciones para reducir polinomios aritméticos. El dominio de estas operaciones constituye la base para la comprensión de otras estructuras matemáticas que se estudia a lo largo de toda la vida estudiantil. Cimentar la práctica de estos conocimientos facilitará los futuros aprendizajes en el área de la matemática. En ésta investigación se probó que el uso de un algoritmo apropiado para realizar operaciones con números reales incide positivamente en el aprendizaje de los estudiantes que ingresan a la Escuela Superior Politécnica de Chimborazo, Facultad de Recursos Naturales, Carrera de Agronomía.

Palabras Claves: Algoritmo, Operaciones, Reales 


\section{Introducción}

El campo de números reales y sus operaciones constituyen un modelo al que la mayoría de teorías matemáticas (complejos, polinomios, funciones, etc.) se ajustan. La comprensión y dominio de este campo constituye el pilar fundamental para el desarrollo de las otras teorías, por esta razón es de vital importancia que los estudiantes que ingresan a la universidad cuenten con fluidez procedimental para realizar operaciones con números reales.

Desde el punto de vista de la matemática, Bermejo et al.(2009) definen un algoritmo como un "método sistemático para resolver operaciones numéricas que consta de un conjunto finito de pasos guiados por reglas que nos permiten sintetizar el cálculo y llegar a un resultado exacto" (p. 194). Por otra parte, las definiciones de las operaciones con números reales permiten plantear algoritmos que tomen en cuenta la jerarquización de operaciones y sus propiedades en la reducción de polinomios aritméticos.

En la República de Ecuador el 35,2\% de los estudiantes de tercero bachillerato tienen un rendimiento insuficiente en la asignatura de matemática, según el Instituto Nacional de Estadísticas y Censos en el Ecuador, Instituto Nacional de Evaluación Educativa (2018); una de las causas es la falta de dominio de las operaciones combinadas (OPC), lo que se evidencia en los estudiantes de la Unidad de Admisión y Nivelación (UAN) de la Escuela Superior Politécnica de Chimborazo (ESPOCH) de la ciudad de Riobamba-Ecuador, Facultad de Recursos Naturales, Carrera de Agronomía. Las dificultades con las que se enfrentan los docentes de matemática de este Nivel de educación son: el desconocimiento de las propiedades de los números reales y la falta de una herramienta que les permita realizar operaciones combinadas entre números reales.

Con el ánimo de resolver este problema se presentó un algoritmo innovador sobre las operaciones entre números reales, que permitió a los estudiantes y docentes resolver polinomios aritméticos comprendiendo y entendiendo tanto lo que se hace como el porqué de ello. La implementación del algoritmo mejoró significativamente los procesos de resolución de las operaciones combinadas en un $65 \%$.

\section{Texto principal:}

Los algoritmos en el proceso de aprendizaje de la matemática permiten que el estudiante cuente con una herramienta para la solución de procesos operacionales; particularmente los algoritmos de las operaciones básicas en el campo de los números reales, desarrollan la destreza del cálculo mental y las referentes a las estructuras del pensamiento lógico matemático; lo que sin duda generará aprendizajes en relación a las operaciones con las diferentes estructuras algebraicas: polinomios, fracciones algebraicas, funciones, etc. El estudiante con dominio de las destrezas operacionales 
relacionará los procesos de las operaciones aprendidas, con las operaciones de los diferentes objetos matemáticos.

El algoritmo de operaciones combinadas con números reales consiste en secuenciar los pasos a seguir en la solución de polinomios aritméticos a través de la jerarquización de operaciones y del análisis previo para discernir cuáles son los pasos del algoritmo a considerar para la solución de ejercicios.

Para la aplicación del algoritmo de solución de operaciones combinadas con números reales es necesario que los estudiantes dominen: las propiedades de los números reales, los procesos de las operaciones básicas y las propiedades de la potenciación y radicación. La teoría de operaciones con números reales se la puede encontrar en Lara (2002).

La siguiente imagen muestra el algoritmo de operaciones combinadas con números que se propuso a la muestra. 


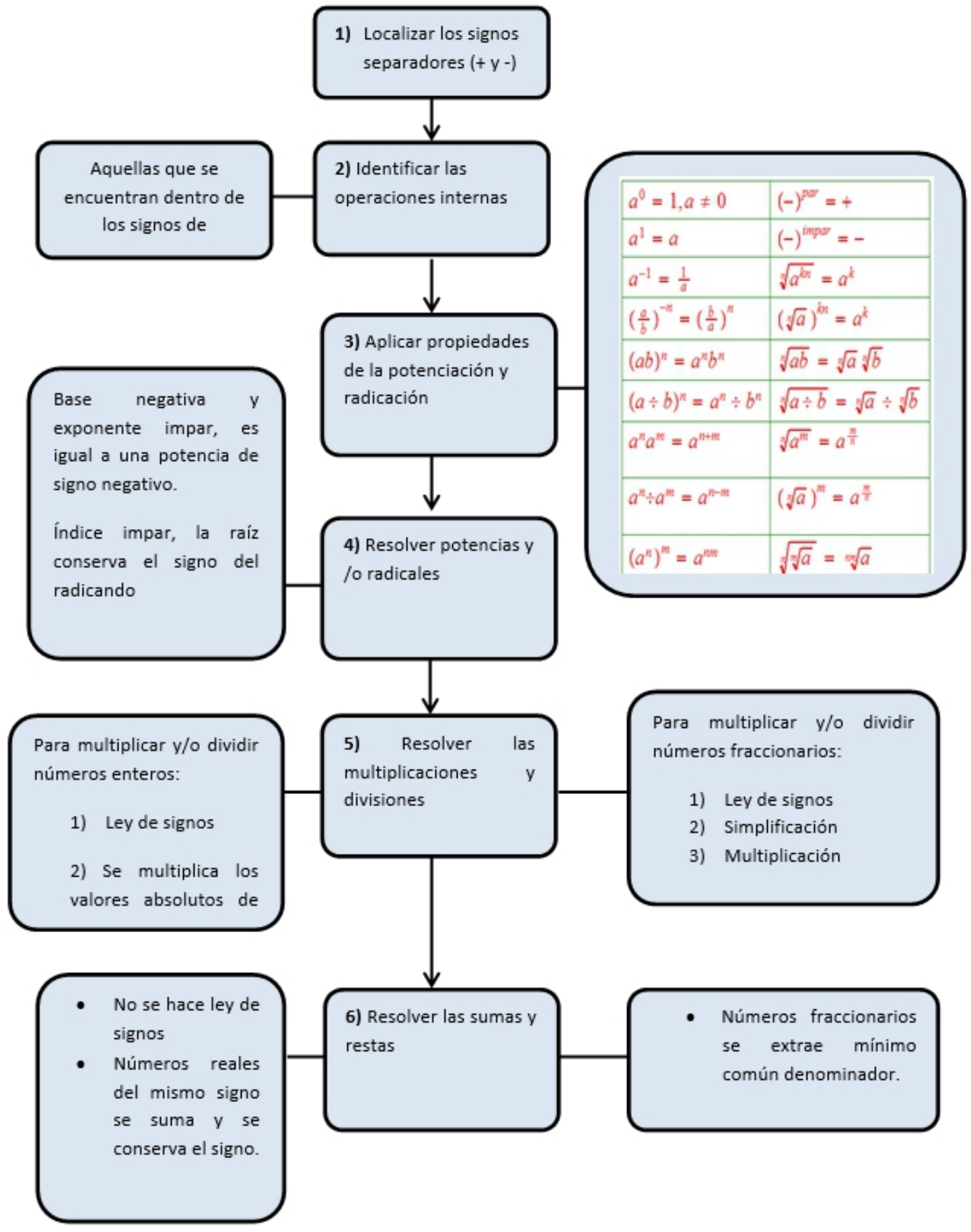

Metodología

La investigación se realizó en la ESPOCH, con estudiantes de la UAN, que eligieron a la facultad de Recursos Naturales, carrera de 
Agronomía, del período Mayo-Agosto de 2018. De los tres paralelos asignados a una de los docentes investigadores, se eligieron al azar dos, uno como grupo de control (18 estudiantes) y el otro como grupo experimental (20 estudiantes). Consecuentemente se tiene:

Población $=60$ estudiantes.

Muestra $=38$ estudiantes.

Inicialmente se aplicó una prueba de diagnóstico (APÉNDICE 1) a los dos grupos, destinada a valorar sus conocimientos respecto a la aplicación de algoritmos para resolver operaciones combinadas con números reales. Con la información obtenida se aplicó un ANOVA para comparar las medias de los puntajes obtenidos en la prueba. También se verificó la validez de la prueba, mediante las pruebas de normalidad, homocedasticidad e independencia de los residuos. Tanto la prueba ANOVA como las pruebas de validación de la misma se realizaron a nivel de significancia del 5\%. Una vez realizadas las pruebas de validez, se comprobó que los residuos no seguían una distribución normal, por lo que se normalizó los datos aplicando el logaritmo natural a los mismos.

Posteriormente, a los dos grupos se les aplicó el modelo experiencial, para el proceso de enseñaza-aprendizaje de las operaciones, propiedades y jerarquización. En la etapa de aplicación del modelo, al grupo experimental se le capacitó en el manejo del algoritmo para resolver OPC entre números reales; mientras que al grupo de control se aplicó la misma metodología sin la utilización del algoritmo como herramienta aprendizaje.

Finalmente, se aplicó a los dos grupos una prueba de salida (APÉNDICE 2); el objetivo de esta prueba fue determinar el nivel de aplicación de los conocimientos relacionados con las operaciones, propiedades y procesos de jerarquización de operaciones con números reales. Con los datos obtenidos se probó que los residuos no siguen una distribución normal y tampoco se pudo normalizar, por lo que se aplicó una prueba no paramétrica para comparar las medias de los puntajes alcanzados por los estudiantes.

\section{Resultados}

\section{Análisis de datos de la prueba de diagnóstico}

Las hipótesis a contrastar son las siguientes:

Ho: Las medias de los rendimientos en la prueba de diagnóstico de los grupos experimental y control son iguales.

Ha: Las medias de los rendimientos en la prueba de diagnóstico de los grupos experimental y control no son iguales.

A continuación se presenta la tabla ANOVA generada por el programa estadístico $\mathrm{R}$, de los datos correspondientes a las notas de la prueba de diagnóstico. 


$\begin{array}{lrrrrr} & \text { Df } & \text { Sum Sq } & \text { Mean Sq } F \text { value } \operatorname{Pr}(>F) \\ \text { rendimiento\$GRUPO } & 1 & 0.265 & 0.2646 & 0.596 & 0.445 \\ \text { Residuals } & 36 & 15.975 & 0.4438 & & \end{array}$

Como se aprecia en la tabla, a un nivel de significancia del 5\% no se rechaza la hipótesis nula de igualdad de medias, por lo que se puede afirmar que los dos grupos, el experimental y el de control presentan las mismas dificultades al resolver operaciones combinadas con números reales.

Gráficamente, se puede apreciar en la siguiente figura, el diagrama de caja de las notas de los grupos experimental y control.

\section{Diagrama de Caja Prueba de Dianóstico}

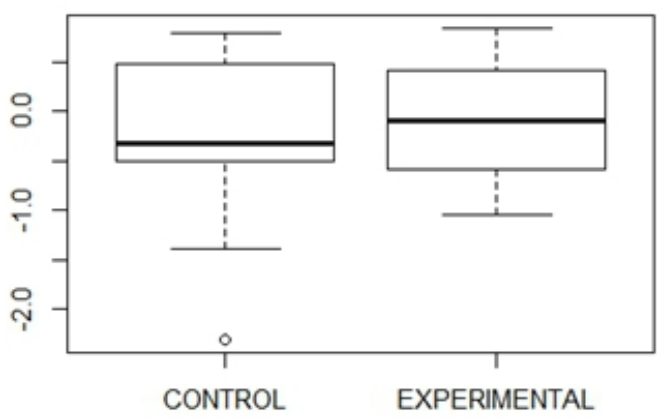

Como se observa en la figura las notas de los dos grupos no difieren significativamente.

En lo que se refiere a la validez de la prueba, se contrastó la normalidad, homocedasticidad e independencia de los residuos.

\section{Normalidad}

A continuación se presenta la prueba de normalidad de Shapiro, generada por el programa estadístico R:

$$
\begin{aligned}
& \text { Shapiro-Wilk normality test } \\
& \text { data: residuals (anova) } \\
& W=0.95266, \text {-value }=0.109
\end{aligned}
$$

Como se observa en el resultado de la prueba de Shapiro, se concluye que los residuos siguen una distribución normal.

Se concluye finalmente que tanto el grupo de control como el grupo experimental están en las mismas condiciones antes de aplicar el tratamiento correspondiente al algoritmo para resolver operaciones combinadas con números reales. 


\section{Homocedasticidad}

Para verificar la homocedasticidad de varianzas se aplicó la prueba de Bartlett, obteniéndose el siguiente resultado:

Bartlett test of homogeneity of variances

data: rendimiento $\$$ RENDIMIENTO by rendimiento $\$$ GRUPO

Bartlett's $\mathrm{K}$-squared $=2.1716, \mathrm{df}=1, \mathrm{p}$-value $=0.1406$

Se observa que no se puede rechazar la hipótesis nula de homogeneidad de varianzas.

\section{Independencia}

Para probar la independencia de los residuos, se aplicó la prueba de Durbin Watson

$$
\text { Durbin-Watson test }
$$

data: rendimiento\$RENDIMIENTO rendimiento\$GRUPO

$$
\mathrm{DW}=2.2055, \mathrm{p} \text {-value }=0.6825
$$

alternative hypothesis: true autocorrelation is greater than $\theta$

Gráficamente se puede observar los supuestos de validación del test.

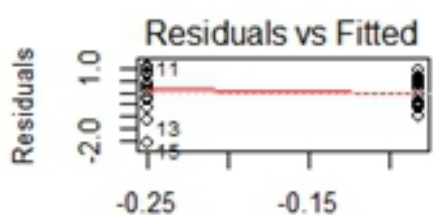

Fitted values

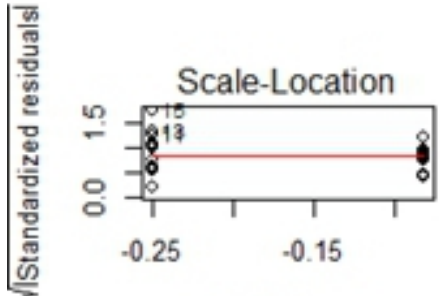

Fitted values

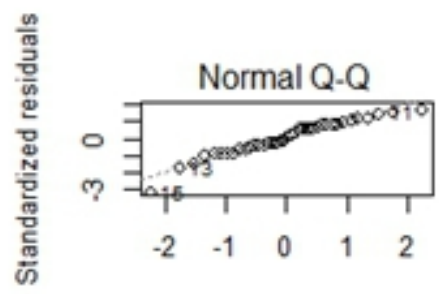

Theoretical Quantiles

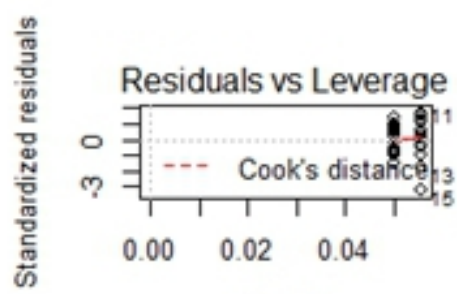

Leverage

Los gráficos parecen indicar que los residuos son aleatorios, independientes, homocedásticos y normales. Lo que se confirmó mediante métodos analíticos.

El gráfico Residuals vs Leverage, indica que no existen puntos con una influencia importante en el cálculo de las estimaciones de los parámetros. 
Se concluye finalmente que tanto el grupo de control como el grupo experimental están en las mismas condiciones antes de aplicar el tratamiento correspondiente al algoritmo para resolver operaciones combinadas con números reales.

\section{Análisis de datos de la prueba de salida}

Ahora las hipótesis a contrastar son:

Ho: Las medias de los rendimientos en la prueba de salida de los grupos experimental y control son iguales.

Ha: Las medias de los rendimientos en la prueba salida de los grupos experimental y control no son iguales.

Como en el caso de la prueba de diagnóstico se presenta el análisis generado por el programa estadístico R.

En primer lugar, se puede apreciar en la siguiente figura, el diagrama de caja de las notas finales de los grupos experimental y control.

Diagrama de Caja Prueba Final

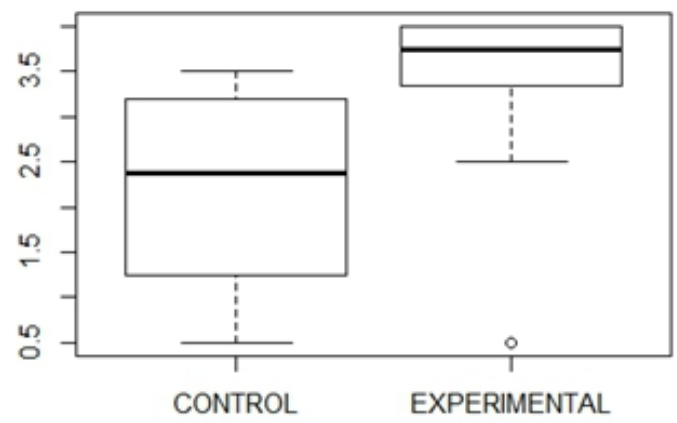

Se observa en la figura que las notas de los dos grupos difieren a favor del grupo que empleó el algoritmo para resolver operaciones combinadas con números reales.

A continuación se observa que los residuos de las notas finales no siguen una distribución normal.

$$
\begin{aligned}
& \text { Shapiro-Wilk normality test } \\
& \text { data: no_parametrico\$CONTROL } \\
& W=0.9056, \text { p-value }=0.07199 \\
& \text { Shapiro-Wilk normality test } \\
& \text { data: no_parametrico } \$ \text { EXPERIMENTAL } \\
& W=0.62666, p \text {-value }=5.634 \mathrm{e}-06
\end{aligned}
$$

Se prueba la igualdad de varianzas: 
$F$ test to compare two variances

data: no_parametrico\$CONTROL and no_parametrico\$EXPERIMENTAL

$\mathrm{F}=1.699$, num $\mathrm{df}=17$, denom $\mathrm{df}=19, \mathrm{p}$-value $=0.2648$

alternative hypothesis: true ratio of variances is not equal to 1

95 percent confidence interval:

0.66186394 .4736893

sample estimates:

ratio of variances

1.699

Se aplica el test de Wilcox para establecer si son o no significativamente diferentes las medias de las notas finales de los dos grupos.

Wilcoxon rank sum test with continuity correction

data: no_parametrico\$CONTROL and no_parametrico\$EXPERIMENTAL $\mathrm{W}=43.5, \mathrm{p}$-value $=6.224 \mathrm{e}-05$

alternative hypothesis: true location shift is not equal to $\theta$

Obsérvese que el p-valor es $6.224 \mathrm{e}-05$, por lo que se rechaza la hipótesis de igualdad de medias de los rendimientos del grupo experimental y de control.

\section{Conclusiones}

- Los grupos de control y experimental presentaron inicialmente las mismas dificultades al resolver operaciones combinadas con números reales.

- Luego de utilizar la herramienta algoritmo para resolver operaciones combinadas con números reales, el grupo experimental demostró mejor capacidad de resolución de tales operaciones respecto al grupo de control.

\section{References:}

1. Bermejo, V. Betancourt, S. y Vela, E. (2009). Cómo enseñar matemáticas para aprender mejor. Madrid: Editorial CCS.

2. Lara, J. \& Arroba, J. (2002). Análisis Matemático. Ecuador: Universidad Central de Ecuador.

3. Instituto Nacional de Evaluación Educativa (2018). Ecuador. Recuperado de http://www.evaluacion.gob.ec/resultados-serbachiller-regimen-costa-2017-2018/

4. Rondero, C. Reyes, A. Acosta, A. (2015). Seguimiento de una innovación curricular: Una asignatura de matemáticas. México. Recuperado de http://eujournal.org/index.php/esj/article/view/5127/4897 


\section{APÉNDICE 1. Prueba de diagnóstico}

Matemática Básica Prueba de diagnóstico

Sírvase desarrollar el siguiente cuestionario. Asegúrese de comprender cada pregunta antes de proceder a su resolución. Registre todo el procedimiento realizado para llegar a su resultado, de lo contrario, el resultado no será válido.

Nombre: Fecha:

1. ¿Conoce usted algún algoritmo para resolver operaciones combinadas entre números reales? En caso afirmativo indique en qué consiste dicho algoritmo.

2. Subraye con una sola línea las operaciones que se deben resolver primero, en el desarrollo del siguiente ejercicio:

$2+4 \cdot 3-5 \sqrt{5+3}-(4-2) \div(3-5)=$

3. Subraye las propiedades de potenciación y radicación que se encuentran en el siguiente ejercicio:

$-2^{3}+\left\{-\sqrt[3]{2^{2}} \cdot \sqrt[3]{2}+7^{100} \div 7^{99}+2(-3+7)^{3}\right\}^{2}$

4. Resuelva los siguientes ejercicios de operaciones combinadas:

$$
\begin{aligned}
& -\left(-\frac{2}{3}\right)^{2}\left(-\frac{2}{3}\right)^{-5}-\sqrt[3]{\sqrt[6]{2^{9}}} \sqrt{2}= \\
& \left(-\frac{2}{3}\right)^{-2}+\left\{\sqrt[3]{2^{-2}} \cdot \sqrt[3]{2^{-4}}-\left[2+5\left(\frac{2}{5}-\frac{1}{15}\right)\right]^{-1}+(-2)^{-3} \cdot(-2)^{5}\right\}=
\end{aligned}
$$




\section{APÉNDICE 2. Prueba de salida}

Sírvase desarrollar el siguiente cuestionario. Asegúrese de comprender cada pregunta antes de proceder a su resolución. Registre todo el procedimiento realizado para llegar a su resultado, de lo contrario, el resultado no será válido.

Nombre:

Fecha:

1. Resuelva los siguientes ejercicios de operaciones combinadas

$-2+5(-3)(4)+\sqrt[3]{8}(-4+5)=$

$-(-2)^{100} \div(-2)^{-102}+\{-[-4+7(-5)]-100 \div(2)\}=$

$\frac{-\frac{3}{2}+\sqrt[3]{1-\frac{7}{8}}}{\left[\frac{4}{5}-2\left(\frac{3}{10}\right)\right]^{-2}}=$

$-\left\{-\frac{6}{5}-\left(-\frac{5}{2}\right)^{-2}-\frac{2}{25}\right\}^{-\frac{1}{2}}=$ 